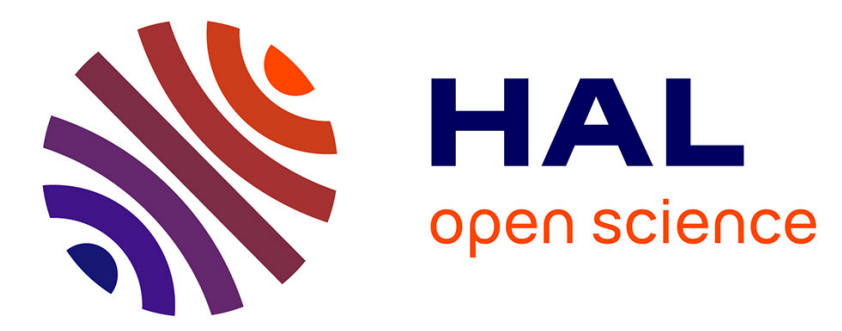

\title{
Magnétisme local du fer dans les superréseaux $\mathrm{Fe} / \operatorname{Ir}(100)$
}

\author{
O. Lemine, S. Andrieu, Ph. Bauer, M. Piecuch
}

\section{To cite this version:}

O. Lemine, S. Andrieu, Ph. Bauer, M. Piecuch. Magnétisme local du fer dans les superréseaux $\mathrm{Fe} / \operatorname{Ir}(100)$. Journal de Physique IV Proceedings, 1996, 06 (C7), pp.C7-207-C7-212. 10.1051/jp4:1996727 . jpa-00254515

\section{HAL Id: jpa-00254515 https://hal.science/jpa-00254515}

Submitted on 1 Jan 1996

HAL is a multi-disciplinary open access archive for the deposit and dissemination of scientific research documents, whether they are published or not. The documents may come from teaching and research institutions in France or abroad, or from public or private research centers.
L'archive ouverte pluridisciplinaire HAL, est destinée au dépôt et à la diffusion de documents scientifiques de niveau recherche, publiés ou non, émanant des établissements d'enseignement et de recherche français ou étrangers, des laboratoires publics ou privés. 


\title{
Magnétisme local du fer dans les superréseaux Fe/Ir(100)
}

\author{
O.M. Lemine, S. Andrieu, Ph. Bauer et M. Piecuch
}

Université Henri Poincaré Nancy 1, URA 155 du CNRS, BP. 239, 54506 Vandouvre-lès-Nancy cedex, France

\begin{abstract}
Résumé: Les propriétés magnétiques du Fer dans les superréseaux $\mathrm{Fe} / \mathrm{Ir}$ (100) sont étudiées par spectrométrie Mössbauer avec $57 \mathrm{Fe}$. Les échantillons, préparés par épitaxie par jet moléculaire, ont un nombre de monocouches de fer ( $57 \mathrm{Fe}$ et/ou $56 \mathrm{Fe}$ ) variant de 3 à 8 et ce pour des épaisseurs en iridium comprises entre 2 et $30 \AA$. . Pour la série à 4 plans où le fer est de structure tétragonale centrée (TC), on observe l'apparition d'un moment magnétique significatif dans les couches centrales de fer au dessus d'un seuil de volume critique, alors que le fer en contact avec l'iridium reste faiblement magnétique. Pour les épaisseurs de fer supérieures à 4 plans, la transition de phase TC - CC contrainte produit de nouveaux sites magnétiques. Une disymétrie marquée entre l'interface $\mathrm{Ir} / \mathrm{Fe}$ inférieure et l'interface $\mathrm{Fe} / \mathrm{Ir}$ supérieure, le long de la direction de croissance, est mise en évidence. Enfin, les effets de relaxation magnétique et le régime d'onde de spin, observés respectivement pour les phases TC et CC contrainte, suggèrent une faible corrélation magnétique de couche à couche.
\end{abstract}

\section{INTRODUCTION}

Il est bien connu que l'épitaxie par jet moléculaire (EJM) permet de synthétiser des phases métalliques nouvelles grâce à des relations d'orientation bien choisies entre le substrat et le dépôt et ce en présence d'un désaccord de maille significatif. Cette technique offre de nouvelles possibilités pour l'étude de la relation existant entre le magnétisme et la structure cristallographique. Dans ce contexte, nous avons pu faire croître du fer pseudomorphe sur $\operatorname{Ir}(100)$ CFC, le désaccord de paramètre étant de l'ordre de $8 \%$. En fait, trois types de superréseaux ont pu être obtenus en fonction du régime de croissance et des épaisseurs relatives de fer - iridium [1] :

i) pour des épaisseurs de fer inférieures à 4-5 monocouches (mc), le mode de croissance est bidimensionnel, ce qui produit des interfaces abruptes au sein de superréseaux uniformément contraints. Le fer est de structure tétragonale centrée (TC), résultant de la déformạtion de la phase CFC pseudomorphe primitive.

ii) pour des épaisseurs de fer et d'iridium respectivement plus grande que 5 et $3 \mathrm{mc}$, on assiste à une transition depuis le régime pseudomorphe vers un régime relaxé. Cette transition ne se produit pas uniformement dans le superréseau mais localement au vu de la disparition des oscillations RHEED, montrant un mode de croissance tridimensionnel en îlots au delà de $5 \mathrm{mc}$ de fer déposées. On obtient une hétérostructure dans laquelle les 4-5 premières mc de fer déposées conservent la structure TC alors que les couches suivantes sont $\mathrm{CC}$ contraintes.

iii) pour les superréseaux à faible épaisseur d'iridium $(\mathrm{mc}<3)$ et d'épaisseur de fer plus grande que $5 \mathrm{mc}$ de fer, l'iridium est contraint par le fer et les propriétés magnétiques de ce dernier sont alors proches de celles du fer $\mathrm{CC}$. Le fer est entièrement relaxé.

Dans le cas du régime pseudomorphe, l'hypothèse d'une contrainte plane uniforme dans tout le superréseau permet l'utilisation d'un modèle élastique simple et l'on a pu montrer que le paramètre a augmente alors que le paramètre $\underline{c}$ diminue pour une épaisseur d'iridium croissante, conduisant à un rapport c/a plus petit que $\sqrt{2}$ ( cas de la structure $C F C$ parfaite). L'augmentation corrélative du volume atomique du fer a été confirmée par des mesures de diffraction $\mathrm{X}$ en incidence rasante, des mesures d'EXAFS et de DAFS. La transition depuis un état faiblement ou non-magnétique vers un état ferromagnétique, observée par les mesures SQUID, a été attribuée à cette variation de volume. Une transition de même nature a été trouvée pour une série de superréseaux à épaisseur d'iridium constante $(15 \AA)$ au dessus d'une épaisseur critique de $3 \mathrm{mc}$ de fer. On ne retrouve la valeur habituelle du moment du fer $\left(2,2 \mu_{\mathrm{B}}\right)$ que pour le régime entièrement relaxé [2]. 
Afin de connaître les propriétés magnétiques locales du fer par le biais des interactions hyperfines mesurées par spectrométrie Mössbauer, nous avons élaboré une série de superréseaux $\mathrm{Fe} / \operatorname{Ir}(100) 15 \AA$ x 20 comprenant tout ou en partie l'isotope ${ }^{57} \mathrm{Fe}$ résonant. L'épaisseur de fer déposée est comprise entre 3 et 8 me.

\section{METHODE EXPERIMENTALE}

Les superréseaux sont élaborés par EJM dans une chambre UHV ayant un vide résiduel inférieur à $10^{-10}$ Torr. Une description plus détaillée de l'élaboration peut être trouvée en [3]. Le fer enrichi à $92 \%$ de l'isotope ${ }^{57} \mathrm{Fe}$ est sublimé par cellule de Knudsen à partir d'une température de $1320^{\circ} \mathrm{C}$ avec une vitesse de croissance de $2.3 \AA \mathrm{A} / \mathrm{min}$. Le superreseau est épitaxié à $100^{\circ} \mathrm{C}$ pour éviter l'interdiffusion. Le nombre de plan déposé est contrôlé par les oscillations RHEED. Deux superréseaux de séquence $1 \mathrm{mc}^{56} \mathrm{Fe}, 2 \mathrm{mc}{ }^{57} \mathrm{Fe}$, $1 \mathrm{mc}^{56} \mathrm{Fe} / \mathrm{Ir} 15 \AA$ et $3 \mathrm{mc}^{56} \mathrm{Fe}, 3 \mathrm{mc}^{57} \mathrm{Fe} / \mathrm{Ir} 15 \AA$ ont été élaborés de façon à pouvoir différencier les différents sites magnétiques observés dans les spectres correspondant aux échantillons entièrement résonants. Dans ce cas, le substrat était maintenu à température ambiante de façon à réduire au minimum l'interdiffusion entre isotopes. Les spectres en géométrie de rétrodiffusion à température ambiante ont été obtenus par détection des électrons de conversion avec un compteur proportionnel à gaz (He mélangé avec $5 \%$ de CH4). Pour les mesures à basses températures $(4,2-290 \mathrm{~K})$, la détection des électrons de conversion a été faite à l'aide d'un multiplicateur d'électrons (galette de micro-cannaux), placé dans un cryostat à hélium liquide [4]. Dans les deux cas, le rayonnement $\gamma$ incident est perpendiculaire au plan de l'échantillon.

\section{RESULTATS ET DISCUSSIONS}

Les spectres à 4,2K, expérimentaux et calculés, sont représentés fig.1(a-d), mettant clairement en évidence une augmentation de l'éclatement hyperfin magnétique pour un nombre $\mathrm{n}$ croissant de $\mathrm{mc}$ de fer. Pour $\mathrm{n}=$ 3, le spectre la ne présente qu'une partie centrale, faiblement éclatée. Un fond large se superpose à la partie centrale dans le spectre $1 \mathrm{~b}$ quand $\mathrm{n}=4$. Pour $\mathrm{n}=5$ et 6 , on constate l'apparition de deux sextuplets Zeeman supplémentaires avec des champs hyperfins différents (spectre 1c). L'abondance relative du sextuplet à raies fines dont le champ hyperfin est le plus grand augmente au détriment de la partie centrale dans le spectre 1d pour $n=8$. En comparaison, le spectre à température ambiante le d'un superréseau avec $15 \mathrm{mc}$ de fer naturel et $3 \mathrm{mc}$ d'iridium (régime entièrement relaxé) ne présente plus de partie centrale ni de fond large. Ce spectre est ajusté avec deux composantes : un sextuplet Zeeman correspondant au fer CC standard et une distribution de champ étroite attribuée au fer en contact avec l'iridium. Cette évolution des spectres Mössbauer semble manifestement liée à l'évolution structurale, depuis le régime pseudomorphe vers le régime relaxé en passant par le régime dit intermédiaire.

\subsection{Le régime pseudomorphe}

Les résultats obtenus pour la série de $4 \mathrm{mc}{ }^{57} \mathrm{Fe} / \mathrm{Ir}(100)$ à différentes épaisseurs d'iridium sont détaillés dans la référence [5]. On peut les résumer comme suit. Les spectres à température ambiante obtenus respectivement en incidence normale et à l'angle magique montrent l'existence de deux composantes paramagnétiques : i) un doublet attribué aux atomes de fer dont le gradient de champ électrique (CGE) est négatif et ii) une raie large reliée aux atomes de fer à faible CGE. De plus, le spectre de l'échantillon $1 \mathrm{mc}^{56} \mathrm{Fe}, 2 \mathrm{mc}^{57} \mathrm{Fe}, 1 \mathrm{mc}^{56} \mathrm{Fe} / \mathrm{Ir}(100) 15 \AA$ a montré que la composante ii) est renforcée, ce qui a permis de l'attribuer sans ambiguîté aux couches centrales du fer de structure TC. La dépendance linéaire du déplacement isomérique envers le volume de la maille TC, calculé à partir du modèle élastique, a corroboré l'hypothèse d'une contrainte uniforme dans les couches de fer et d'iridium déformant le fer CFC pseudomorphe. Enfin, pour des épaisseurs en Ir inférieures à $4,7 \AA ̊$, les spectres à $4,2 \mathrm{~K}$ sont similaires à celui de la fig. 1a alors que pour les épaisseurs supérieures, on obtient des spectres comme celui de la fig. $1 \mathrm{~b}$.

La partie grisée de la fig. $1 \mathrm{~b}$ représente le spectre expérimental et calculé de l'échantillon $1 \mathrm{mc}^{56} \mathrm{Fe}, 2 \mathrm{mc}^{57} \mathrm{Fe}, 1 \mathrm{mc}^{56} \mathrm{Fe} / \mathrm{Ir}(100) 15 \AA$ à $4,2 \mathrm{~K}$. On en déduit que seules les deux mc de fer centrales (composante ii) ci dessus) ressentent un champ magnétique hyperfin Bhf significatif, alors que celles en contact avec l'irridium conservent un champ faible. L'ajustement du spectre $1 \mathrm{~b}$ a été conduit de la façon suivante : 


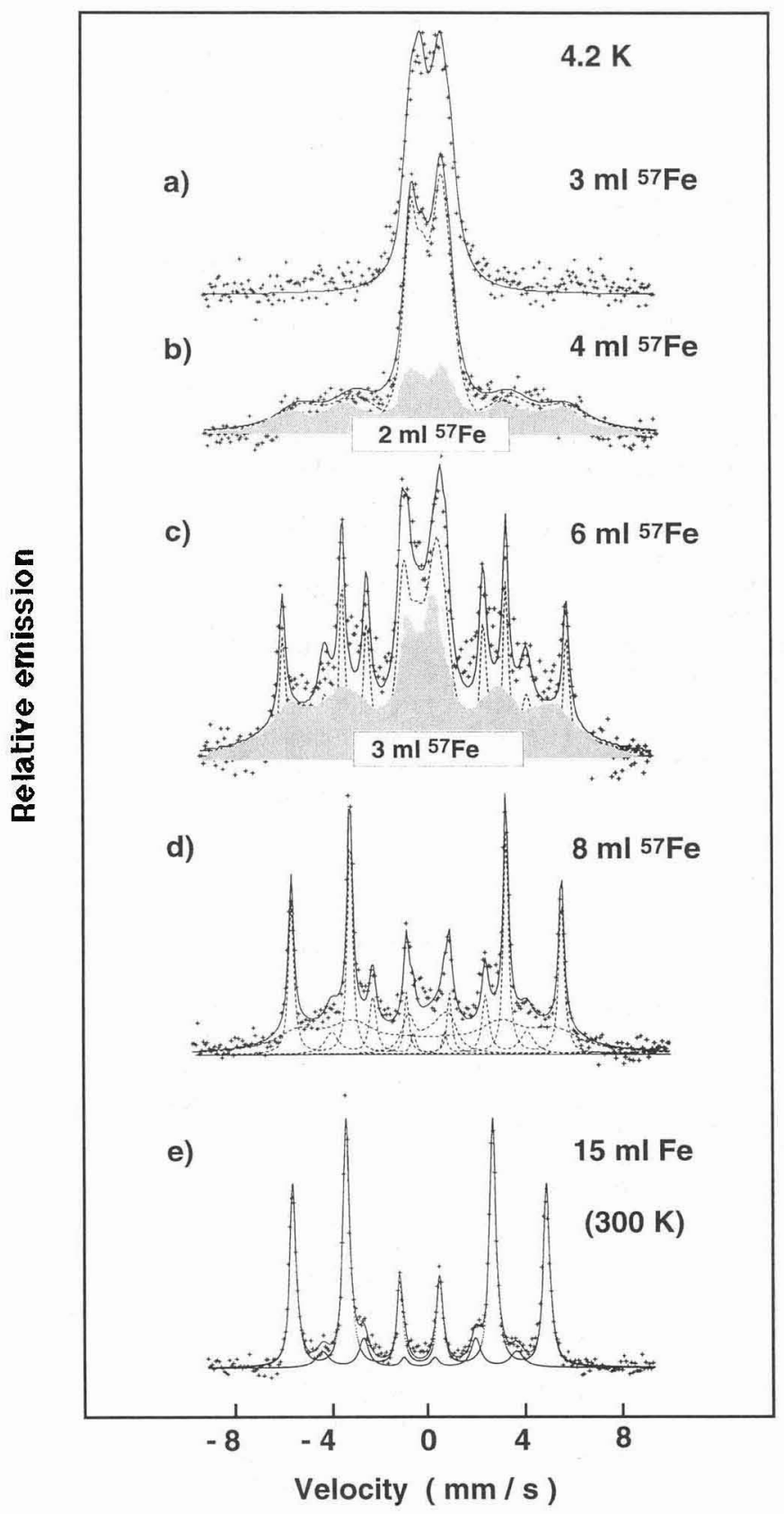

Figure 1: Spectres expérimentaux $(4,2 \mathrm{~K})$ et calculés de superréseaux de n monocouches ${ }^{57} \mathrm{Fe} / \mathrm{Ir}(100)$ avec $n=3(\mathrm{a}), \mathrm{n}=4$ (b), $\mathrm{n}=6$ (c), $\mathrm{n}=8$ (d), en comparaison avec le spectre à température ambiante de l'échantillon 15 couchesFe / $3 \AA \operatorname{Ir}(100)$ (e). La partie grisée de (b) et (c) correspond aux spectres expérimentaux et calculés des échantillons ${ }^{56} \mathrm{Fe}, 2 x^{57} \mathrm{Fe},{ }^{56} \mathrm{Fe}$ et $3 x^{57} \mathrm{Fe}, 3 x^{56} \mathrm{Fe}$ de même épaisseur en iridium. 
-la partie centrale i) (55\%) reliée aux atomes de fer en contact avec l'iridium est calculée avec l'Hamiltonien non diagonal où les angles $\beta$ et $\theta$ (angles respectifs entre le rayonnement $\gamma$ et les axes de GCE et de champ hyperfin) doivent être spécifiés. Dans l'hypothèse d'un rayonnement $\gamma$ quasi perpendiculaire à la surface de l'échantillon, on trouve $\beta=9^{\circ}, \mathrm{e}^{2} \mathrm{qQ} / 2=-0.30 \mathrm{~mm} / \mathrm{s}$ (en accord avec le dépouillement des spectres à $300 \mathrm{~K}$ ), $\theta=50^{\circ}$ et $\mathrm{Bhf}=4,8 \mathrm{Tesla}$. On en déduit d'une part que l'axe de GCE est pratiquement perpendiculaire au plan des couches de fer c'est à dire parallèle à l'axe de symétrie quaternaire, et d'autre part qu'une anisotropie magnétique perpendiculaire apparaît pour le fer en contact avec l'iridium. Le même type d'ajustement a été effectué pour la partie centrale du spectre 1a : l'axe de GCE est en moyenne incliné de $30^{\circ}$ par rapport à la normale au plan. On peut supposer que pour $3 \mathrm{mc}$ de fer, la présence de marches ou de terrasses aux interfaces influe de façon significative sur la symétrie locale. Pour les deux échantillons, les chămps hyperfins de 3,5 (1a) et 4,8 (1b) Tesla doivent être comparés à celui que l'on trouve dans le cas des alliages $\mathrm{Fe}_{\mathrm{x}} \mathrm{Ir}_{1-\mathrm{x}} \mathrm{CFC}$ antiferromagnétiques pour $0,3<\mathrm{x}<$ $0,65[5]$.

- le fond résonant (45\%) attribué aux atomes des deux mc de fer centrales est caractérisé par un important élargissement des raies d'émission. Une large distribution de champ reliée à une variation continue de l'environement local des atomes de fer semble exclue ici. De ce fait, nous avons supposé l'existence d'un phénomène de relaxation magnétique, source d'élargissement important des raies spectrales. Le fond resonant a été calculé avec un modêle simple dans lequel on considère que pendant la transition entre niveaux nucléaires il se produit un renversement entre deux états de spin électronique $S_{z}=$ $+1 / 2$ et $S_{Z}=-1 / 2$. La fréquence de basculement rendant compte de l'élargissement se situe aux voisinage de $12 \mathrm{MHz}$ pour un champ Bhf de l'ordre de 35 Tesla. Il est remarquable que l'apparition d'un moment magnétique de $2 \mu_{\mathrm{B}}$ fluctuant ne concerne que les deux mc de fer TC centrales au dessus du seuil critique de 4,7 $\AA$ d'iridium. Ce résultat est en accord avec les mesures d'aimantation qui montrent l'apparition d'un moment global significatif pour le même seuil critique [2]. Cependant, ce moment moyen est très petit quand on le compare à celui obtenu à partir du champ hyperfin des deux couches de fer TC centrales, même si l'on suppose l'existence de deux couches interfaciales magnétiquement mortes. Ce désaccord peut s'expliquer par le phénomène de relaxation magnétique invoqué ci-dessus.

\subsection{Le régime intermédiaire}

La partie grisée de la fig. 1c représente le spectre expérimental et calculé de l'échantillon $3 \mathrm{mc}^{57} \mathrm{Fe}, 3 \mathrm{mc}^{56} \mathrm{Fe}$ / Ir(100) superposé à celui de l'échantillon avec $6 \mathrm{mc}$ de ${ }^{57} \mathrm{Fe}$. On en déduit immédiatement que les pics fins supplémentaires du spectre de l'échantillon entièrement résonant ne peuvent provenir que des $3 \mathrm{mc}$ de fer supérieures (dans le sens de la croissance). Le calcul du spectre grisé reproduit celui de la fig. $1 \mathrm{~b}$ avec les paramètres $\mathrm{Bhf}=5 \mathrm{Tesla}, \mathrm{e}^{2} \mathrm{qQ} / 2=-0,30 \mathrm{~mm} / \mathrm{s}, \theta=43^{\circ}$ (anisotropie perpendiculaire), sauf que le rapport $\mathrm{Fe}$ interfacial / Fe TC est de 1 à 2 au lieu de $1 / 1$. Les abondances relatives calculées pour le spectre 1c montrent que l'on obtient une hétérostructure dans le sens de la croissance avec $3 \mathrm{mc}$ de fer TC suivies de $3 \mathrm{mc}$ de fer parmi lesquelles $2 \mathrm{mc}$ en moyenne sont caractérisées par les champs respectifs de 36,2 et 25,8 Tesla. Au sein des deux sextuplets Zeeman les intensités relatives sont dans le rapport 3:4:1;1:4:3, montrant que l'aimantation des atomes de fer concernés est confinée dans le plan des couches. Dans le cas de l'échantillon à $8 \mathrm{mc}$ de fer, la partie centrale du spectre $1 \mathrm{~d}$ est réduite; le même calcul que ci-dessus conduit à une séquence composée en moyenne d'une $\mathrm{mc}$ de fer en contact avec l'iridium suivie de $3 \mathrm{mc}$ de fer TC présentant des phénomènes de relaxation magnétique, puis de $3 \mathrm{mc}$ de fer avec un champ de 34,6 Tesla et d'une mc avec un champ de 25 Tesla. Ce dernier champ hyperfin est comparable à celui du sextulpet minoritaire du spectre de la fig.1e : on peut l'identifier à celui des atomes de fer en contact avec l'iridium dans la structure $C C$ relaxée. Ansi, nous avons mis en évidence un régime intermédiaire pour lequel la structure TC subsiste jusqu'à 3-4 mc de fer dans le sens de la croissance, alors que les couches de fer supérieures sont dans la structure CC contrainte. Il est à souligner que dans cette structure contrainte la valeur du champ hyperfin dépasse largement celle du fer CC standard (36,2 contre 34,2 Tesla).

Finalement nous avons étudié l'évolution thermique du superréseau à $8 \mathrm{mc}$ de Fe dans le domaine de température 4,2 - 300K. Les spectres de la fig. 2 montrent clairement la différence de comportement des composantes magnétiques. 


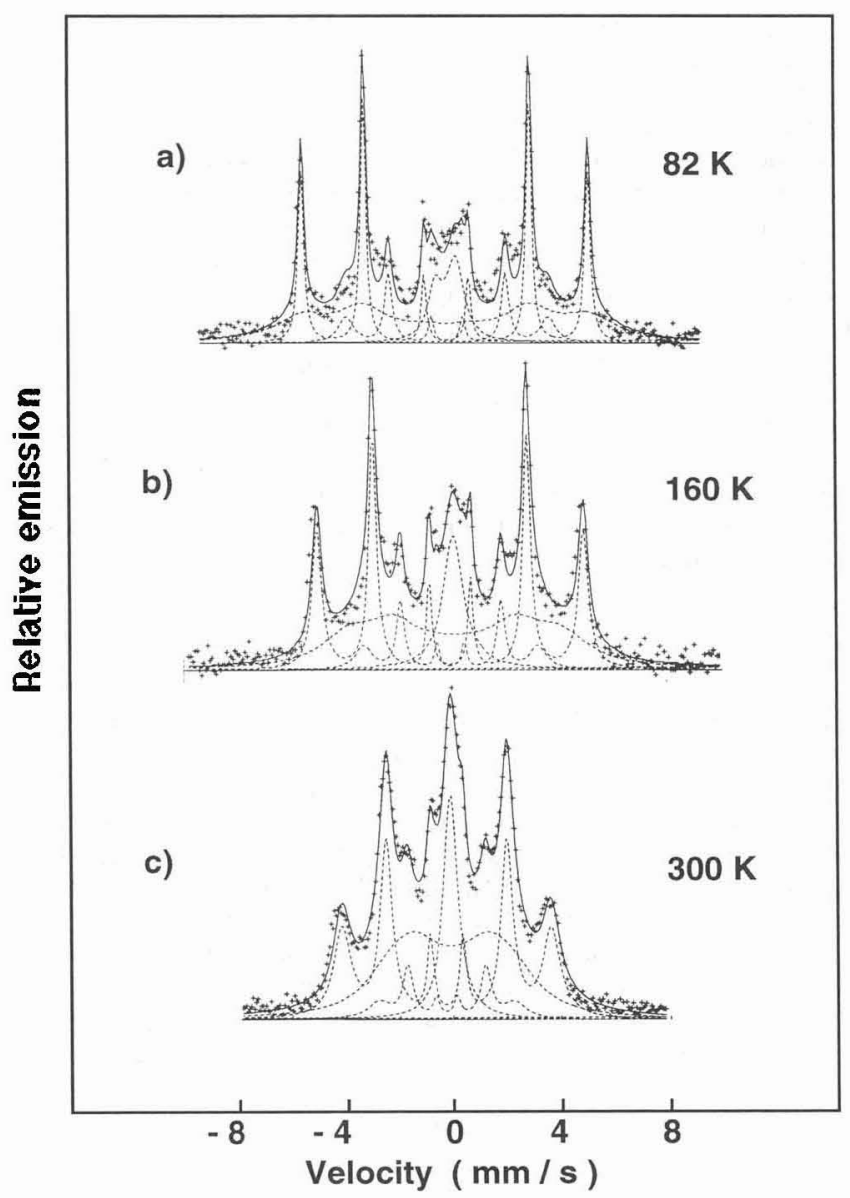

Figure 2 : Evolution thermique de l'échantillon $8 \mathrm{x}^{57} \mathrm{Fe} / 15 \AA \operatorname{Ir}(100): 82 \mathrm{~K}$ (a), $160 \mathrm{~K}$ (b) et $300 \mathrm{~K}$ (c).

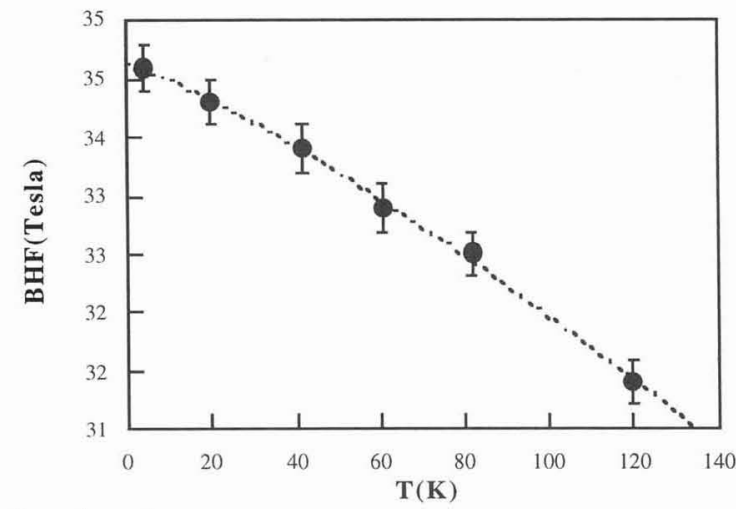

Figure 3 : Variation en fonction de la températre du champ hyperfin Bhf correspondant au sextuplet Zeeman le plus abondant de l'échantillon $8 x^{57} \mathrm{Fe} / 15 \AA \operatorname{Ir}(100)$. La courbe calculée représente la fonction $\mathrm{Bhf}(\mathrm{T}) / 34,62=1-210^{-4} \mathrm{~T}^{1,24}$ 
A $120 \mathrm{~K}$, l'interface Ir/Fe inférieure devient paramagnétique, alors que les phénomènes de relaxation relatifs au fer TC sont plus marqués. A partir de $160 \mathrm{~K}$ les raies du sextuplet Zeeman principal s'élargissent à leur tour (fig.2b). A la température ambiante (fig.2c), l'interface Fe/Ir supérieure a un champ hyperfin de 15,6Tesla, beaucoup plus petit que celui de l'interface de l'échantillon à $15 \mathrm{mc}$ de fer, entièrement relaxé (fig.1.e). Dans le domaine de température 4,2-160K les raies d'emission du sextulpet Zeeman principales demeurent fínes, ce qui permet d'avoir une bonne détermination de la variation de Bhf en fonction de la température. Cependant, la loi habituelle $\mathrm{Bhf}(\mathrm{T}) / \mathrm{Bhf}(0)=1-\mathrm{BT}^{3 / 2}$ caractérisant un mode d'onde de spin standard conduit à $\mathrm{Bhf}(0)$ inférieur à $\mathrm{Bhf}(4,2)$. Le meilleur ajustement est représenté sur la fig.3, conduisant à une loi en puissance 1,24 pour $T$ avec $B h f(0)=34,62$ Tesla et un facteur $B=210^{-4} \mathrm{~K}^{-1,24}$.

Ce résulat montre que le comportement des ondes de spin dans les couches de fer $\mathrm{CC}$ contraintes supérieures diffère notablement de celui que l'on observe pour un ferromagnétique ordinaire, le coefficient $\mathrm{B}$ étant à peu près 100 fois plus important que dans le cas du fer massif [6]. Il est remarquable que dans le cas des superréseaux $3 \mathrm{mcFe}(100) / \mathrm{Ag}$ (111), pour des épaisseurs en argent comprises entre 4 et $20 \mathrm{mc}$, une valeur comparable de $\mathrm{B}$ ait été déterminée dans ce système caractérisé par un comportement ferromagnétique bidimensionnel (loi linéaire en $T$ ) [6]. Il ressort que l'existence de phénomènes de relaxation magnétique dans la phase de fer $\mathrm{TC}$ et de modes mous d'onde de spin pour la phase $\mathrm{CC}$ contrainte tend à montrer une faible corrélation de couche à couche dans les superréseausx Fe / Ir (100).

\section{Conclusion}

Les propriétés magnétiques locales des superreseaux Fe / $\operatorname{Ir}(100) 15 \AA$ ont été étudiées par Spectrométrie Mössbauer pour des épaisseurs de fer comprises entre 3 et 8 monocouches. Dans le régime pseudomorphe, pour 4 plans de fer, les deux couches centrales de fer tétragonal centré présentent un champ hyperfin élevé ( 35 Tesla) avec un phénomène de relaxation magnétique, alors que les atomes en contact avec l'iridium sont caractérisés par un champ hyperfin faible (5 Tesla). Dans le régime intermédiaire, pour 6-8 plans de fer, le profil magnétique dans le sens de la croissance montre l'évolution depuis la structure tétragonale centrée (interface Ir/Fe inférieure) vers la structure cubique centrée contrainte (interface Fe/Ir supérieure). L'évolution thermique de la composante à fort champ (34,6 Tesla) reliée au fer cubique centré contraint montre un régime d'onde de spin différent de celui d'un ferromagnétique normal. Ce fait, associé au phénomène de relaxation magnétique observé pour la phase tetragonale centrée, suggère une faible corrélation magnétique de couche à couche le long de la direction de la croissance.

\section{Réferences}

[1] S. Andrieu, F. Lahatra Razafindrasima, E. Snoeck, H. Renevier, M. Brunel and M. Piecuch, Phys.Rev. B 52 (1995) 9938.

[2] S. Andrieu, M. Piecuch, L. Hennet, J. Hubsch and E. Snoeck, Europhys.lett. 26 (1994) 189.

[3] Ph. Bauer and G. Marchal, Proc. SIF, edited by I. Ortalli, Vol 50, (Editrice Compositori, Bologna) $1996, \mathrm{p} 895$.

[4] Ph. Bauer, S. Andrieu and M. Piecuch, Il Nuovo Cimento 18D (1996) 299-304.

[5] M. Shiga, Phys. Stat. Sol. (b) 43 (1971) K 37.

[6] C. J. Gutierrez, Z. Q.Qui, H.Tang, M. D. Wieczorek, S. H. Mayer and J. C. Walker, Phys.Rev.B 44 (1991) 2190-2197. 